\title{
Effect of needle size on cancer detection, pain, bleeding and infection in TRUS-guided prostate biopsies: a prospective trial
}

\author{
Michael McCormack, MD, FRCSC;, Alain Duclos MD, FRCSC;, Mathieu Latour MD, FRCPC; ; Marie Hélène \\ McCormack, BSc, ${ }^{\ddagger}$ Daniel Liberman, MD, CM; ${ }^{*}$ Orchidee Djahangirian, MD; Josette Bergeron, RN, ${ }^{\xi}$ \\ Luc Valiquette, MD, FRCSC,; Kevin Zorn, MD, FRCSC ${ }^{*}$
}

*Division of Urology, Department of Surgery, Université de Montréal, Hôpital Saint-Luc du CHUM, Montreal, QC; 'Department of Pathology, Université de Montréal, Hôpital Saint-Luc du CHUM, Montreal, QC; ; FECC Montreal, Montreal, QC; \$Hôpital Saint-Luc du CHUM, Montreal, QC

Cite as: Can Urol Assoc J 2012;6(2):97-101. http://dx.doi.org/10.5489/cuaj.11169

\section{Abstract}

Introduction: Transrectal ultrasound (TRUS)-guided prostate biopsies using 18G calibre needles are widely used; most often 12-core tissue samples of the peripheral zone are obtained. Although the diagnostic yield of prostate biopsies is fair, there is still a potential for false negative results, which necessitates repeat biopsies. In an effort to improve the accuracy of prostate biopsies, different sampling schemes have been developed. One strategy has been to increase the number of core biopsies performed on each patient. Another strategy has been to improve the reliability of prostate biopsies using larger calibre needles, thereby increasing the amount of tissue obtained for each core biopsy.

Methods: After approval by our institutional review board, we prospectively compared two biopsy needle sizes (18G vs. 16G) in relation to prostate cancer diagnosis, pain, bleeding and infection rates on 105 patients. Each patient underwent 6 TRUS-guided prostate biopsies with the standard 18G needle and 6 other biopsies with the experimental 16G needle. To evaluate possible complications related to the use of a larger 16G needle in the experimental group, we compared pain, bleeding and infection rates with a control group of 100 patients who underwent 12 biopsies with a single 18G needle (18G group). Pain, bleeding assessment and infection events were evaluated using patient questionnaires and telephone interviews.

Results: TRUS-guided prostate biopsies using 16G calibre needles did not increase cancer detection or non-malignant pathology rate, including prostatic intraepithelial neoplasia (PIN) and atypical small acinar proliferatio (ASAP). Pain, bleeding and infectious complications were similar in both groups. Infection was defined as temperature above $38^{\circ} \mathrm{C}$ occurring within 48 hours after the procedure. We identified 4 patients with post-biopsy fever in the experimental (16/18G) group and 4 other patients in the (18G) control group. The post-biopsy infection rate is higher than reported just a few years ago and indicates that quinolone resistant Escherichia coli seems to be more prevalent in our urban setting than previously suspected. Limitations to our study include small group numbers. Conclusion: Larger 16G needles appear to be safe for TRUS-guided prostate biopsies. Further study in a larger, multi-institutional, prospective, randomized manner with $16 \mathrm{G}$ needles is warranted to assess the theoretical benefit of larger core biopsies in prostate cancer detection.

\section{Introduction}

Transrectal ultrasound (TRUS)-guided prostate biopsies using $18 \mathrm{G}$ calibre needles is widely used. Systematic prostate sampling is performed and most often 12-core tissue samples of the lateral peripheral zone are obtained during each series of biopsies. ${ }^{1}$

Different sampling schemes have been developed to improve the accuracy of prostate biopsies. One strategy has been to increase the number of core biopsies performed on each patient. Initially, sextant biopsies were commonly employed but studies have shown that about 30\% of significant cancers are missed with this sampling strategy. ${ }^{2}$ Therefore, sextant biopsies have been replaced by extended core biopsies. ${ }^{1,2}$

Another strategy to improve the reliability of prostate biopsies is to use larger calibre needles, which increase the amount of tissue obtained for each core biopsy. This could potentially improve histological sampling, thereby enhancing the accuracy of prostate cancer diagnosis. Small increases in prostate needle diameter can yield significant increases in tissue sample volume. The mean core volume of $16 \mathrm{G}$ needles is almost twice that of $18 \mathrm{G}$ needles. ${ }^{3}$ However, any increase in the prostate biopsy needle calibre could potentially increase pain, bleeding and infection rate.

We evaluated cancer detection and complications including pain, bleeding and infection rates in patients undergoing prostate biopsies with larger 16G calibre needles versus the widely used $18 \mathrm{G}$ needle.

\section{Methods}

After approval by our institutional review board, we prospectively compared two biopsy needle sizes (18G vs. 16G) in relation to prostate cancer diagnosis, pain, bleeding and 
McCormack et al.

infection rates on 105 patients. Patients involved in this study were referred exclusively by urologists to our academic hospital outpatient clinic. Our clinic performs over 1500 TRUSguided biopsies per year. All biopsies were performed by three skilled operators. Pathological analysis was done by a single pathologist in our institution.

Patients were placed in a lateral decubitus position, with the knees and hips flexed 90-degrees, to perform TRUSguided biopsies. The application of a topical anesthetic agent ( $1 \%$ lidocaine gel) was administered into the rectum. Anesthesia was also achieved with the administration of $10 \mathrm{cc}$ of plain lidocaine (1\%) injected at the junction between the seminal vesicle and the prostate (peri-prostatic nerve block). Transrectal ultrasound and digital rectal examination (DRE) were performed to confirm previous findings by referring physicians. An automatic Pro-mag 2.2 device (ProMag, MD Tech, Gainesville, FL) was used for all biopsies. Needles used were the Angiotech 18G $\times 20 \mathrm{~cm}$ (model $\# 763818200$ ) and the Angiotech $18 \mathrm{G} \times 20 \mathrm{~cm}$ (model \#765016200).

We recruited 105 patients for the experimental (18G/16G) group. In this cohort, each patient underwent 6 lateral peripheral zone (PZ) TRUS-guided prostate biopsies with the standard 18G needle and six adjacent lateral PZ biopsies with the experimental $16 \mathrm{G}$ needle. Thus, lateral peripheral prostate zones were assessed with both needles in each patient in each sextant zones (Fig. 1). This tissue sampling strategy allowed us to establish cancer detection rates for each needle size in every patient. The inclusion and exclusion criteria are outlined in Table 1. A single pathologist in our teaching hospital reviewed all biopsies. McNemar's non-parametric test was used for statistical analysis.

To evaluate possible complications related to the use of a larger 16G needle in the experimental group, we compared pain, bleeding and infection rates with a control group of 100 patients who underwent 12 lateral PZ biopsies with a single $18 \mathrm{G}$ needle (18G group) performed by the same 3

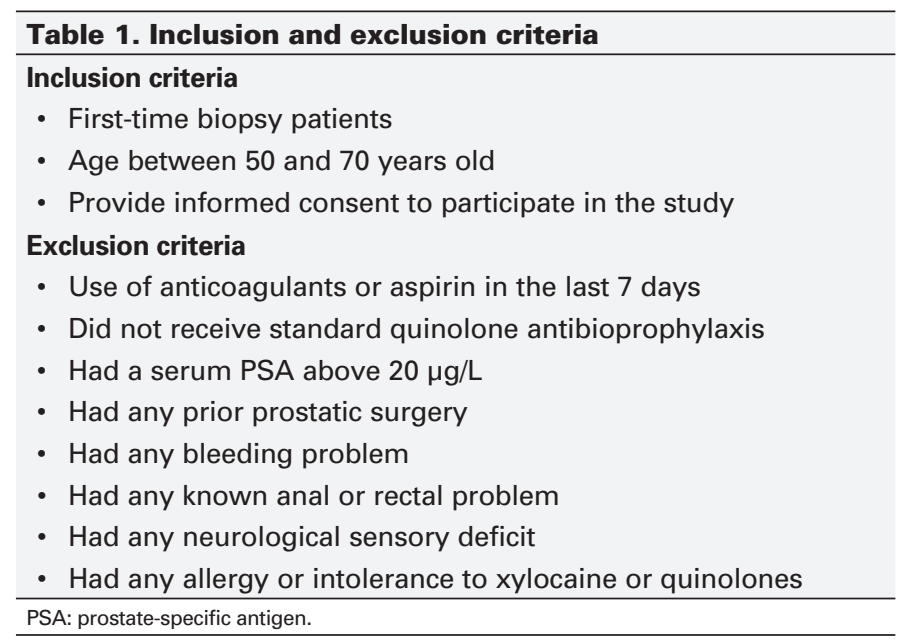

urologists. Pain, bleeding assessment and infection events were evaluated using the same methods in both groups. A single clinical nurse throughout the study administered patient questionnaires and telephone interviews to minimize procedural variability.

Pain was assessed in both groups with a validated visual analogue scale (VAS). ${ }^{4}$ Pain was evaluated 15 minutes following the procedure and by phone interview one and three weeks later. An independent samples $t$ Test was used for statistical analysis. Bleeding was assessed in both groups with a questionnaire 15 minutes after the procedure and by phone interview one and three weeks later. Patients were questioned on the presence of blood in their urine, stools or sperm. A chi-square test was used for statistical analysis.

All patients received standard quinolone antibioprophylaxis based on the referring physician's preference. Either one- or three-day quinolone regimens prescribed by the referring urologists were accepted. Both one- or three-day regimens using an oral fluoroquinolone 30 to 60 minutes before biopsy have been shown to be equivalent in relation to septic protection. ${ }^{5-9}$ For each patient we verified that the antimicrobial prophylaxis was taken in accordance with the Canadian Urological Association guidelines and the American Urological Association Best Practice Policy Statement on Urologic Surgery. ${ }^{10,11}$

Infection was defined as temperature above $38^{\circ} \mathrm{C}$ occurring within 48 hours after the procedure. All patients were instructed to proceed to an emergency room to receive wide spectrum intravenous antibiotics if they had fever after the biopsies. Infection rate was assessed by phone interview by a clinical nurse. Efforts were done to obtain microbiological results of the patients with fever who consulted emergency hospitals for intravenous antibiotic treatment.

We did not use cleansing enemas before the biopsies, due to its cost and inconvenience; moreover, there is no evidence supporting their use. ${ }^{10}$

\section{Results}

Transrectal ultrasound and DRE were performed on each patient. In the experimental 18G/16G group, the mean PSA was $5.36 \mu \mathrm{g} / \mathrm{L} \pm 2.23$ (range: $0.5-12.6$ ), and the mean volume was $47.81 \mathrm{~mL} \pm 19.64$ (range: $15.5-144.7$ ).

Prostate cancer detection rate was determined by using both needles (16G and 18G) in each patient in the experimental group. Study patients (105) underwent 6 biopsies with a 16G core size needle and 6 other biopsies with a 18G core size needle. Therefore, 630 biopsy specimens were available for pathological analysis for each needle size. Prostate cancer was diagnosed in $36.2 \%$ (38 cases) of $18 \mathrm{G}$ needle specimens and in $31.4 \%$ (33 cases) of $16 \mathrm{G}$ needle specimens. The difference was not statistically significant between both groups $(p=0.33)$. 
Effect of needle size on cancer detection, pain, bleeding and infection

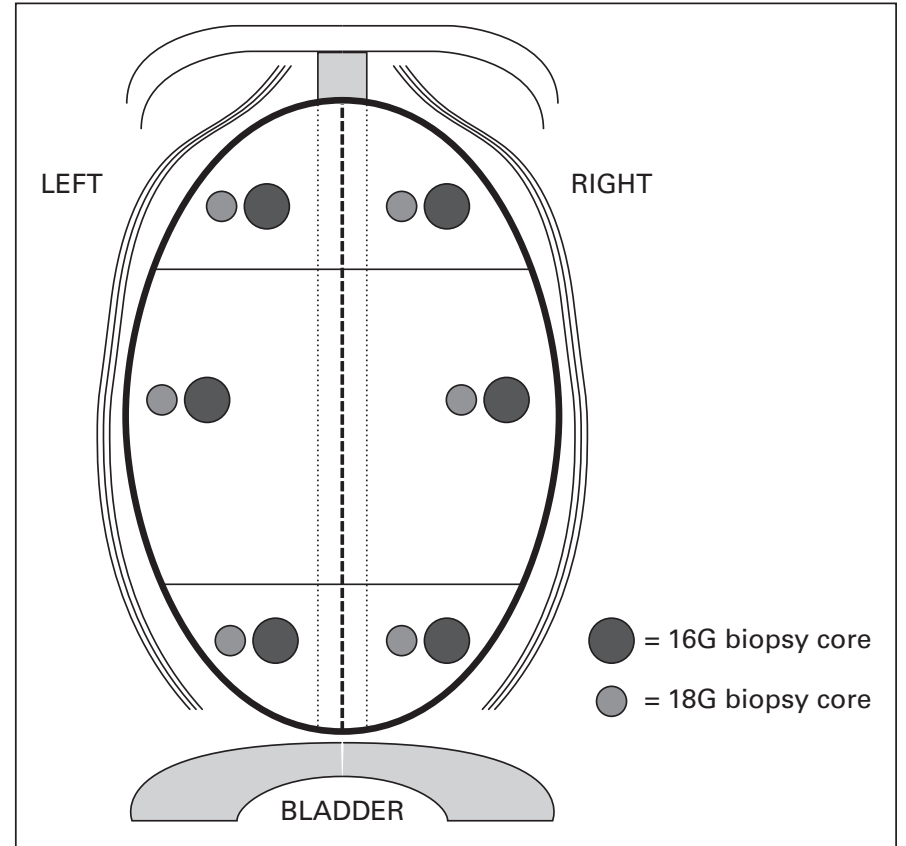

Fig. 1. Illustration of the lateral peripheral prostate zones assessed with both needles in each patient.

Further pathological analysis revealed prostatic intraepithelial neoplasia (PIN) in $21.9 \%$ (23 cases) of the $18 \mathrm{G}$ and in $26.7 \%$ (28 cases) of the 16G specimens, respectively. We also observed atypical small acinar proliferation (ASAP) in $8.6 \%$ (9 cases) of the $18 \mathrm{G}$ and in $12.4 \%$ (13 cases) of the 16G specimens, respectively. There was no statistical difference in PIN detection $(p=0.44)$ or in ASAP detection $(p=0.42)$ with the $16 \mathrm{G}$ or the $18 \mathrm{G}$ needle size.

Pain was equivalent both experimental (18G/16G) and control (18G) groups. At 15 minutes, the mean VAS score was $0.74 \pm 1.33$ (range: 0-6) compared to the experimental group, $0.58 \pm 1.23$ (range: $0-5)(p=0.38)$. At 7 days postintervention, the mean pain score was $1.03 \pm 1.86$ (range: $0-8$ ) in the experimental group and $1.19 \pm 2.09$ (range: $0-8.5)$ in the control group $(p=0.57)$. At three weeks, no patients reported pain.

Furthermore, bleeding was equivalent in both groups. At 15 minutes post- biopsy, $10.5 \%$ (11) of patients in the experimental group and $13 \%$ (13) of patients in the control group reported the presence of blood. One week after the procedure $81.6 \%$ (84) of patients in the experimental group and $92 \%$ (92) in the control group reported the presence of blood. Overall, there was no statistical difference between the experimental and the control group regarding the presence of blood after 15 minutes $(p=0.35)$ or after 7 days $(p=0.11)$.

The infection rate was equivalent in the experimental and control groups. Post-biopsy fever over $38^{\circ} \mathrm{C}$ occurred in $4 \%$ (4) of the control group and 3.8\% (4) of the experimental group. Thus, increasing needle size from 18G to $16 \mathrm{G}$ did not affect the infection rate. Efforts were made to obtain microbiological results of patients who developed fever after the procedure. Quinolone resistant Escherichia coli was found in 4 patients ( 2 patients in each group). Four patients with fever had negative urine cultures. In summary, a $4 \%$ infection rate was present in both groups of patients. In our study, no septic patients required catheterization.

\section{Discussion}

TRUS-guided prostate biopsies using 18G calibre needles are widely used and most often 12-core tissue samples of the peripheral zone are obtained. Although the diagnostic yield of prostate biopsies is fair, there is still a potential for false negative results and repeat biopsies are often required. Indications for repeat biopsy may include previous negative biopsies in patients with an elevated PSA or an abnormal DRE, the presence of nonmalignant pathology, such as ASAP or multifocal high-grade PIN and active surveillance for prostate cancer.

To improve the accuracy of prostate biopsies, different sampling schemes have been developed. One strategy has been to increase the number of core biopsies performed on each patient. Initially sextant biopsies were commonly employed, but studies have shown that about $30 \%$ of significant cancers are missed with that sampling strategy. ${ }^{1,2} \mathrm{~A}$ meta-analysis regrouping 87 studies with a total of 20698 patients has shown that sampling schemes with 12 cores detected 30\% more cancers than the sextant scheme. Therefore, sextant biopsies have been replaced by extended core biopsies. ${ }^{1,2}$

Increasing the number of biopsies over 12 ("saturation biopsies") has also been proposed. However sampling schemes ranging from 18 to 24 cores did not detect significantly more cancers on initial biopsy procedures and were associated with a higher incidence of complications. ${ }^{12,13}$ Thus saturation biopsies are not presently recommended for initial screening, but may be useful in patients with previous negative biopsies and persistently elevated or rising PSA levels.

Another strategy to improve the reliability of prostate biopsies is to use larger calibre needles, which increase the amount of tissue obtained for each core biopsy. This could potentially improve histological sampling, thereby enhancing the accuracy of prostate cancer diagnosis. Larger needle sizes have been studied in breast cancer; it was found that the quantity and quality of breast biopsy specimens depend on the needle size. ${ }^{14}$ Of the three needle sizes tested (14G, 16G and 18G), 14G biopsy needles had the best quality of breast biopsy specimens. ${ }^{14}$

We set out to evaluate cancer detection and complications including pain, bleeding and infection rates in patients undergoing prostate biopsies with larger $16 \mathrm{G}$ calibre needles 
McCormack et al.

versus the $18 \mathrm{G}$ needle.

There are few reports concerning the use of $16 \mathrm{G}$ needles for prostate biopsies. Pain has been evaluated in patients undergoing prostate biopsies with 16G needles. A prospective, randomized trial was undertaken with both a VAS and a facial expression scale (FES) in patients undergoing transperineal prostate biopsies. This study did not reveal any significant difference in pain perception. ${ }^{4}$ Pain has also been evaluated in patients undergoing TRUS-guided prostate biopsies using 16G calibre needles. A prospective study involving 103 patients undergoing biopsies with a 16G needle showed no significant differences in the VAS pain scores when compared to 101 patients undergoing biopsies with the a $18 \mathrm{G}$ needle. ${ }^{15}$ Although both these studies failed to reveal better cancer detection rates, the authors argue that 16G needles improve specimen quality and could lead to better cancer detection. To our knowledge, no study has been published evaluating bleeding rates in patients undergoing 16G prostate biopsies.

In our study, prostate cancer detection rate was determined in each patient of the experimental group by using both needles (16G and 18G). Each patient underwent 6 TRUS-guided prostate biopsies with the standard 18G needle and another 6 biopsies with the experimental 16G needle. Lateral peripheral prostate zones were assessed with both needles in each patient. Therefore 630 18G-core and 630 16G-core biopsy specimens were available for pathological analysis. This tissue sampling strategy allows us to establish cancer detection rates for each needle size in every patient. Statistical analysis did not reveal any significant difference in the pathological findings between the 16G and the 18G biopsy specimens. Both 16G and 18G sampling yielded similar results for prostate cancer, PIN and ASAP detection.

To evaluate possible complication rates related to the use of larger 16G needles in the experimental group, we compared pain, bleeding and infection rates with a control group of 100 patients who underwent 12 standard biopsies with a single 18G needle. Pain and bleeding questionnaires were used 15 minutes following the procedure and by phone interview 1 and 3 weeks later. There was no statistical difference in pain or bleeding rates between both groups. We found that using 16G or 18G needles is not associated with any difference in pain or bleeding.

Infection was defined as temperature above $38^{\circ} \mathrm{C}$ occurring within 48 hours after the procedure. We identified 4 patients with post-biopsy fever in the experimental (16/18G) group and 4 other patients in the (18G) control group. Once again there was no significant difference between both groups. Thus, increasing the needle size from 18G to 16G did not affect the infection rate. Efforts were made to obtain microbiological results of patients who developed fever following the procedure. Quinolone resistant $E$. coli was found in 2 patients in the (16/18G) group and in 2 patients in the control (18G) group. Four patients with fever had negative cultures. Overall our study shows a $4 \%$ infection rate in both groups of patients.

A $4 \%$ post-biopsy infection rate is higher than reported just a few years ago and indicates that quinolone resistant E. coli seems to be more prevalent in our urban setting than previously suspected. ${ }^{16}$ Although none of our patients developed any major complications from their post-biopsy infections, such complications have been reported and may be serious. New antibiotic regimens should be developed for TRUS-guided prostate biopsies.

\section{Conclusion}

TRUS-guided prostate biopsies using 16G calibre needles did not increase cancer detection or non-malignant pathology rate, including ASAP and PIN. Pain, bleeding and infectious complications were similar in both groups. Post-biopsy infection rate is higher than reported just a few years ago and indicates that quinolone resistant $E$. coli seems to be more prevalent in our urban setting than previously suspected. New antibiotic regimens should be developed for TRUS-guided prostate biopsies. Limitations to our current study include small group numbers. Nevertheless, the use of larger 16G needles appears to be safe for TRUS-guided prostate biopsies. Further study in a larger, multi-institutional, prospective, randomized manner is warranted to assess the theoretical benefit of larger core biopsies in prostate cancer detection.

Competing interests: None declared.

This paper has been peer-reviewed.

\section{References}

1. Eichler K, Hempel S, Wilby J, et al. Diagnostic value of systematic biopsy methods in the investigation of prostate cancer: a systematic review. J Urol 2006;175:1605-12. http://dx.doi.org/10.1016/ S0022-5347(05)00957-2

2. Benway BM, Andriole GL. Prostate biopsy, UpToDate, June 16, 2011. http://www.uptodate.com/ contents/prostate-biopsy. Accessed March 6, 2012.

3. Saredi G, Sighinolfi MC, Fidanza F, et al. Does needle calibre affect pain and complication rates in patients undergoing transperineal prostate biopsy? A prospective, randomized trial. Asian J Androl 2009;11:67882. Epub 2009 Sep 21. htrp://dx.doi.org/10.1038/aja.2009.43

4. Price DD, McGrath PA, Rafii A, et al. The validation of visual analogue scales as ratio scale measures for chronic and experimental pain. Pain 1983;17:45-56. http://dx.doi.org/10.1016/03043959(83)90126-4

5. Zani EL, Clark OA, Rodrigues Netto N Jr. Antibiotic prophylaxis for transrectal prostate biopsy. Cochrane Database Syst Rev 2011;5:CD006576.

6. Schaeffer AJ, Montorsi F, Scattoni V, et al. Comparison of a 3-day with a 1-day regimen of an extended-release formulation of ciprofloxacin as antimicrobial prophylaxis for patients undergoing transrectal needle biopsyof the prostate. BJU Int 2007;100:51-7. http://dx.doi.org/10.1111/i.1464410X.2007.06848.x 
Effect of needle size on cancer detection, pain, bleeding and infection

7. Sabbagh R, McCormack M, Peloquin F, et al. A prospective randomized trial of 1-day versus 3-day antibiotic prophylaxis for transrectal ultrasound guided prostate biopsy. Can I Urol 2004;11:2216-9.

8. Kapoor DA, Klimberg IW, Malek GH, et al. Single-dose oral ciprofloxacin versus placebo for prophylaxis during transrectal prostate biopsy. Urology 1998;52:552-8. hitp://dx.doi.org/10.1016/500904295(98)00296-9

9. Aron M, Rajeev TP, Gupta NP. Antimicrobial prophylaxis for transrectal needle biopsy of the prostate: a randomized controlled study. BJU Int 2000:85:682-5

10. El-Hakim A, Moussa S. CUA guidelines on prostate biopsy methodology. Can Urol Ass J 2010;4;89-94.

11. AUA Best Practice Policy Statement on Urologic Surgery Antimicrobial Prophylaxis. (2008) (Reviewed and validity confirmed 2011.) (http://www.auanet.org)

12. Stewart CS, Leibovich BC, Weaver AL, et al. Prostate cancer diagnosis using a saturation needle biopsy technique after previous negative sextant biopsies. J Urol 2001;166:86-91. http://dx.doi.org/10.1016/ S0022-5347(05)66083-1

13. Pepe P, Aragona F. Saturation prostate needle biopsy and prostate cancer detection at initial and repeat evaluation. Urology 2007;70:1131-5. http://dx.doi.org/10.1016/i.urology.2007.07.068
14. Helbich TH, Rudas M, Haitel A, et al. Evaluation of needle size for breast biopsy: comparison of 14- 16and 18-gauge biopsy needles. AJR Am J Roentgenol 1998;171:59-63.

15. Inal GH, Oztekin VC, Uğurlu 0 , et al. Sixteen gauge needles improve specimen quality but not cancer detection rate in transrectal ultrasound-guided 10-core prostate biopsies. Prostate Cancer Prostatic Dis 2008;11:270-3. Epub 2008 Jun 3. http://dx.doi.org/10.1038/pcan.2008.34

16. Shigehara K, Miyagi T, Nakashima T, et al. Acute bacterial prostatitis after transrectal prostate needle biopsy: clinical analysis. J Infect Chemother 2008;14:40-3. Epub 2008 Feb 24. http://dx.doi. org/10.1007/s10156-007-0570-3

Correspondence: Dr. Michael McCormack, Associate Professor of Surgery, Division of Urology, Department of Surgery, Université de Montréal, Hôpital Saint-Luc du CHUM, 1058, rue Saint-Denis, Montreal, QC H2X 3J4; m.mccormack@umontreal.ca 